\title{
Influence of collateral water drinking on bar pressing under complex reinforcement contingencies'
}

EVALYN F. SEGAL ${ }^{2}$ AND WILLIAM M. BANDT

SAN DIEGO STATE COLLEGE

Rats were run on 2-link chained schedules. The first link was always FI 2 min.; the second link was either FR 10; DRL $10 \mathrm{sec}$; or Concurrent FR 10 DRL $10 \mathrm{sec}$. All responding was on a single bar. Responding in the first link was reinforced by onset of the second-link stimulus, and in the second link by $45 \mathrm{mg}$ Noyes food pellets. When the ad lib water bottle was removed from the chamber, rats on Chain FI FR or Chain FI DRL showed an increase in FI bar presses but no change in second-link behavior; rats on Chain FI Concurrent FR DRL showed the major change in the second link; DRL behavior was almost wholly replaced by FR behavior.

Clark (1962) noted that water drinking prevented typical performance on a VI food schedule. We extend Clark's observations by showing the effect of allowing or preventing drinking on some complex performances. Method

Adult, albino rats, two males and two females, were kept at $80 \%$ of ad lib weight by food, but not water, deprivation. One of each sex was assigned to Chain FI 2 min. Concurrent FR 10 DRL $10 \mathrm{sec}$; a male to Chain FI $2 \mathrm{~min}$. FR 10; and a female to Chain FI 2 min. DRL $10 \mathrm{sec}$. First links were marked by flashing of a cue lamp; second links by steady illumination of the lamp. A cycle of the chain consisted of one completion of the FI 2 min. contingency followed by 11 food reinforcements in the second link. Sessions for males were 20 cycles long; for the smaller females, 14 cycles.

The conditioning chamber contained only one bar, mounted centrally on the front wall; the cue lamp above it; the water bottle to the right of it; and the food cup to the left of it. All but the front wall and floor were made of transparent lucite. The chamber was housed in a darkened, sound-resistant room containing white masking noise.

All schedule components were standard except for the Concurrent FR DRL. During this schedule, the FR and DRL contingencies held simultaneously. Every bar press spaced $10 \mathrm{sec}$. or more from the last was reinforced via the DRL contingency; every 10 th press spaced less than $10 \mathrm{sec}$. from the last was reinforced via the FR contingency. Every reinforcement reset both contingencies.

The rats were pretrained on smaller FI, FR, and DRL requirements before the present values were put into effect. The data reported here were from the 18th and 19 th days under the present conditions. On the 18 th day the water bottle was removed before sessions began; on the 19th day, it was replaced.

Results

Figure 1 shows the results of removing water. Day 19, rather than 17 , is offered as the control (with water) day because of recording difficulties on day 17 .

Chain FI 2 min. Concurrent FR 10 DRL $10 \mathrm{sec} .:$ Rat RF. When water was present (day 19) FI pressing was at a rate barely sufficient to advance the chain from the first to the second link; there was no FI "scalloping." RF took its food entirely via the FR contingency in the first 2 cycles of day 19 and entirely via the DRL contingency in the remaining cycles. Removing water (day 18) increased the rate and curvature of FI responding, and transformed the second-link performance to mainly FR behavior.

Rat RM. Control performance (day 19) included a large amount of FI pressing with marked scalloping; and an irregular alternation of DRL and FR behavior in the second link. Removing water (day 18) increased FI responding but, as in $\mathbf{R F}$, had its main effect on secondlink behavior, which was transformed to mainly FR behavior.
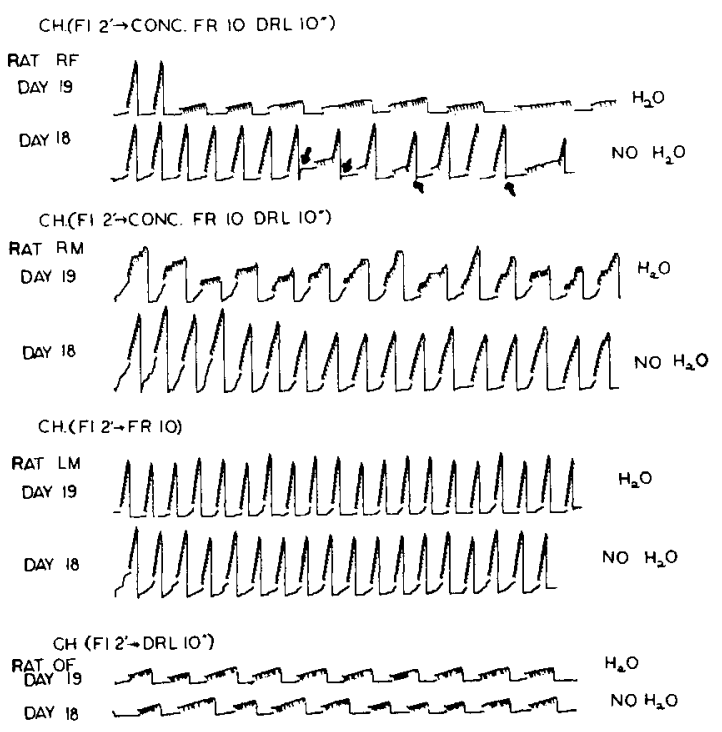

Fig. 1. Cumulative bar presses for the first $60 \mathrm{~min}$. of day 18 (without water) and 19 (with water). The pen reset to baseline after every 11 food reinforcements, and was offset slightly downward and to the right during first (FI 2 min.) links. Momentary downward pen deflections during second links mark food arrivals. 
Chain FI 2 min. FR 10: Rat LM. Control performance (day 19) showed very shallow FI scallops and typically high and steady FR rates. Removing water (day 18) increased the steepness of the FI scallops but had no apparent effect on FR behavior.

Chain FI 2 min. DRL 10 sec.: Rat OF. Control performance (day 19) showed a very low FI rate, with virtually no scalloping. DRL performance was at the low and steady rate typical of this schedule. Removing water (day 18) led to a very slight increase in the number of FI responses, but still without scalloping. There was no evident effect on DRL behavior.

\section{Discussion}

This experiment antedated most of those on psychogenic polydipsia earlier reported from this laboratory (e.g., Deadwyler \& Segal, 1965) and records of water drinking were not made. We will reconstruct the control pattern of drinking by inference from the effects of withholding water, together with recollections of some visual observations.

1. The conditions for drinking. Drinking seemed to occur whenever the prevailing schedule included interval contingencies. Thus, we infer that all rats drank during FI links, with a consequent great or small depression of bar pressing, for all rats pressed more when drinking was prevented. Rats drank during Concurrent FR DRL, again with a depression of pressing, for both rats on this schedule pressed much faster when drinking was prevented. In contrast, we infer that drinking did not occur during the FR schedule, for preventing drinking did not increase $F R$ rate.

2. The role of drinking in timing. Neither did preventing drinking increase DRL response rate, and yet direct observation showed that the rat (OF) did drink between presses when water was available. The explanation of this anomaly is very likely the DRL contingency. The rat had never been reinforced for any but low-rate pressing; so, unlike the other three rats, it had no alternative but to go on pressing at low rate, even when water was absent.

3. Rat RM: direct observations. The arrival of the first food pellet of a cycle generally led to drinking. The rat visited first the food cup, and ate, and then the water bottle, and drank, and then the bar, and pressed. If this sole press produced another pellet, the sequence repeated. If it did not, the rat's posture changed: it grabbed the bar in both paws, one above and one below, and jiggled it rapidly until a pellet arrived. Thus, the contingencies appear to have generated two response sequences in this rat, each under discriminative control. The basic sequence was one press-eat-drink-one presseat-drink ... The second, conditionally inserted into the basic sequence whenever a single press failed to produce food, was vigorous two-pawed jiggling of the bar.

4. Interactions of drinking and bar pressing. Only where the reinforcement contingencies provided a wholly different option, viz., in the concurrent schedule, did the prevention of water drinking cause a dramatic change in the character of bar pressing. The effect on FI performance of preventing drinking was to cause more nearly typical FI behavior to appear. The effect on simple FR or DRL performances was nil. Although the performances were more or less different depending on whether drinking was allowed or not, it would seem a mistake to conclude that the "dry" performance was a truer reflection of the reinforcement contingencies than the "wet" one, or that drinking competed with "normal" bar pressing. Rather, it seems that collateral behavior such as drinking is as much a normal outcome of certain reinforcement contingencies as are the familiar bar-pressing patterns.

5. A note on inductive effects. FI response rate seemed to reflect whatever rate was reinforced in the second, food link. Thus, the DRL schedule conditioned low rate, and so OF emitted a low rate in the FI link, too. The FR schedule conditioned high rate, and so LM emitted a relatively high rate in the FI link. The concurrent schedule generated a predominantly low rate in RF and so its FI rate was low; it generated a much higher rate in RM and so its FI rate was much higher. These inductive effects, moreover, were independent of water drinking.

\section{References}

Clark, F. C. Some observations on the adventitious reinforcement of drinking under food reinforcement. J. exp. Anal. Behav., 1962, $5,61-63$.

Deadwyler, S, A., \& Segal, Evalyn F. Determinants of polydipsia: VII. Removing the drinking solution midway through DRL sessions. Psychon. Sci., 1965, 3, 185-186.

\section{Notes}

1. Supported by NSF G 18132, NSF GB 1605, and NIMH 8505 . 2. On leave at the Royal College of Surgeons of England. Send reprint requests to Mr. David Oden, Department of Psychology, San Diego State College, San Diego, California, 92115. 